\title{
INDEX FOR VOLUME 113
}

Armitage, D. H. \& Nelson, C. S. A harmonic quadrature formula characterizing open strips

Armitage, D. H., Gardiner, S. J. \& Netuka, I. Separation of points by classes of harmonic functions.

Auinger, K. The word problem for the bifree combinatorial strict regular semigroup .

Baker, J. W. \& Lau, A. T. Compact left ideal groups in semigroup compactification of

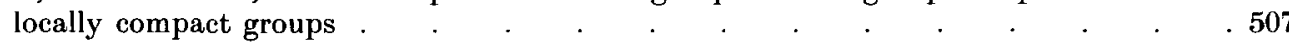

Baxter, M. Symmetry characterizations of certain distributions. 3. Discounted additive functionals and large deviations for a general finite-state Markov chain _ . . 381

Boston, N. \& Ullom, S. V. Representations related to CM elliptic curves . . . . $\quad$. 71

Brown, B. M., Kirby, V. G., Evans, W. D. \& Plum, M. Safe numerical bounds for the Titchmarsh-Weyl $m(\lambda)$-function . . . . . . . . . . . . . . . . 583

Cariñena, J., Martinez, E. \& Sarlet, W. Geometric characterization of separable second-

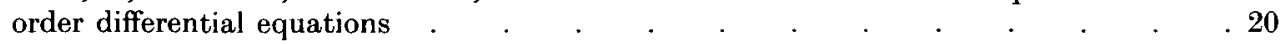

Castillo, J. M. F. \& Sánchez, F. Upper $l_{p}$-estimates in vector seqence spaces, with some

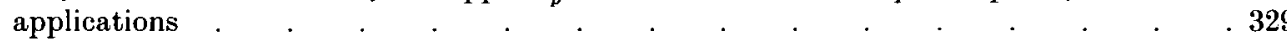

Dales, H. G. \& Millington, A. Translation-invariant linear operators _ . . . 161

Diaz, J. C. \& Miñarro, M. A. On Fréchet Montel spaces and their projective tensor product 335

Donkin, S. Invariant functions on matrices. . . . . . . . . . . . . . . . . . 23

Drury, S. W. \& Guo, K. Some remarks on the restriction of the Fourier transform to surfaces. . . . . . . . . . . . . . . . . . . . . . . . . 153

Edmunds, D. E., Opic, B. \& Pick, L. Poincaré and Friedrichs inequalities in abstract Sobolev spaces

Evans, M. J. T-systems of certain finite simple groups. $\quad$. . . . . . . . . .

Evans, W. D., Plum, M., Brown, B. M. \& Kirby, V. G. Safe numerical bounds for the Titchmarsh-Weyl $m(\lambda)$-function . . . . . . . . . . . . . . . . . . $\quad .583$

Farr, G. E. A generalization of the Whitney rank generating function _ . . $\quad . \quad 267$

Gardiner, S. J., Netuka, I. \& Armitage, D. H. Separation of points by classes of harmonic functions.

Gibson, C. G \& Hobbs, C. A. Simple singularities of space curves . . . . $\quad . \quad 297$

Green, D. J. On the cohomology of the sporadic simple group $J_{4} \quad$. $\quad . \quad$. $\quad . \quad 253$

Guo, K. \& Drury, S. W. Some remarks on the restriction of the Fourier transform to

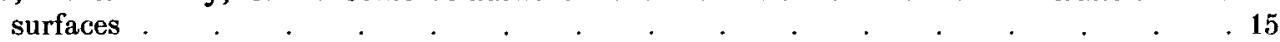

Hennings, M. A. Strongly spin-preserving solutions of the Yang-Baxter equation and their

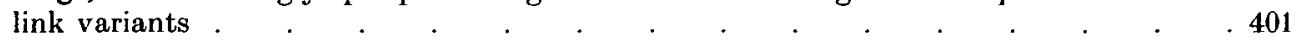

Higgins, P. M. Combinatorial results for semigroups of order-preserving mappings $\quad$. 281

Hinkkanen, A. Julia sets of rational are functions uniformly perfect . . . . . . 543

Hobbs, C. A. \& Gibson, C. G. Simple singularities of space curves . . . . . . . 297

Hobson, D. G. \& Rogers, L. C. G. Recurrence and transience of reflecting Brownian

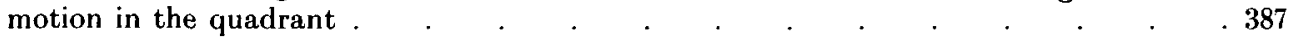

Hofmann, K. H. \& Morris, S. A. Generators on the arc component of compact connected

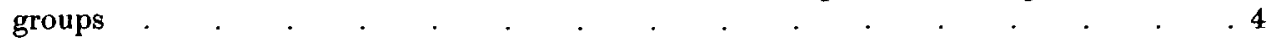

Holland, D. \& Wilson, S. M. J. Localization and class groups of module categories with

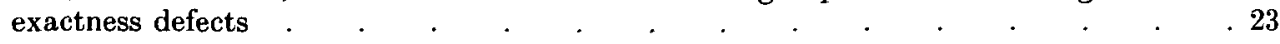

Johnson, F. E. A. A rational invariant for certain infinite discrete groups . $\quad . \quad$. 473 


\section{Index}

Kamiya, S. On $H$-balls and canonical regions of loxodromic elements in complex hyperbolic

Kesten, H. Convergence in distribution of lightly trimmed and untrimmed sums are equivalent

Kiesel, R. Power series methods and almost sure convergence

Kirby, V. G., Evans, W. D., Plum, M. \& Brown, B. M. Safe numerical bounds for the Titchmarsh-Weyl $m(\lambda)$-function

Lau, A. T. \& Baker, D. W. Compact left ideal groups in semigroup compactification of locally compact groups .

Maeda, H. A criterion for a smooth surface to be Del Pezzo

Martinez, E., Cariñena, J. \& Sarlet, W. Geometric characterization of separable secondorder differential equations

Majid, S. Transmutation theory and rank for quantum braided groups . . . . . . 45

Mbekhta, M. Semi-Fredholm perturbations and commutators . . . . . . . . 173

Millington, A. \& Dales, H. G. Translation-invariant linear operators _ . . . . 161

Miñarro, M. A. \& Díaz, J. C. On Fréchet Montel spaces and their projective tensor product 335

Morris, S. A. \& Hofmann, K. H. Generators on the arc component of compact connected groups

Nelson, C. S. \& Armitage, D. H. A harmonic quadrature formula characterizing open strips

Netuka, I., Armitage, D. H. \& Gardiner, S. J. Separation of points by classes of harmonic functions.

Opic, B., Pick, L. \& Edmunds, D. E. Poincaré and Friedrichs inequalities in abstract Sobolev spaces

Oxley, J. \& Whittle, G. On sign-representable matroids _ . . . . . . . . . . . . . . . . 499

Pan, Y. $L^{2}$ estimates for convolution operators with oscillating kernels $\quad . \quad$. . . $\quad .179$

Pick, L., Edmunds, D. E. \& Opic, B. Poincaré and Friedrichs inequalities in abstract Sobolev spaces

Plum, M., Brown, B. M., Kirby, V. G. \& Evans, W. D. Safe numerical bounds for the Titchmarsh-Weyl $m(\lambda)$-function

Rickert, J. H. Simultaneous rational approximations and related diophantine equations $\quad 461$

Robinson, P. L. Quasifree representations of Clifford algebras _ . . . . . . . . . . 487

Roach, G. F. \& Zhang, B. Spectral representation and scattering theory for the wave equation with two unbounded media . . . . . . . . . . . . . . . . . 423

Rogers, L. C. G. \& Hobson, D. G. Recurrence and transience of reflecting Brownian motion in the quadrant. . . . . . . . . . . . . . . 387

Sánchez, F. \& Castillo, J. M. F. Upper $l_{p}$-estimates in vector sequence spaces, with some applications

Sander, J. W. On primes not dividing binomial coefficients . . . . . . . . . . . . $\quad .225$

Sarlet, W., Martinez, E. \& Cariñena, J. Geometric characterization of separable secondorder differential equations

Schwärzler, W. \& Welsh, D. J. A. Knots, matroids and the Ising model . . . . 107

Slack, M. Infinite loop spaces with trivial Dyer-Lashof operations _ . . . . . . . . . 311

Spanoudakis, N. K. Operators in finite distributive subspace lattices, I . . . . 141

Sugie, J. \& Yoneyama, T. On the Liénard system which has no periodic solutions . . 413

Swinnerton-Dyer, Sir Peter. The Brauer group of cubic surfaces _ . . . . . . . . . 449

Szücs, A. Double point surfaces of smooth immersions $M^{n} \rightarrow \mathbb{P}^{2 n-2} \quad$. . . . . . . . 601

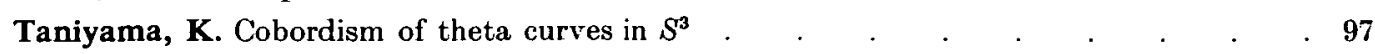

Teragaito, M. Roll-spun knots . . . . . . . . . . . . . . . . . . . . . 91

Ullom, S. V. \& Boston, N. Representations related to CM elliptic curves . . . . . . 71 


\section{Index}

Urbanski, M. \& Zamboni, L. On free actions of $\Lambda$-trees $\quad$. $\quad$. . . . . . . . $\quad .535$

Velani, S. L. Diophantine approximation and Hausdorff dimension in Fuchsian groups $\quad 343$

Welsh, D. J. A. \& Schwärzler, W. Knots, matroids and Ising model . ‘ . . . . 107

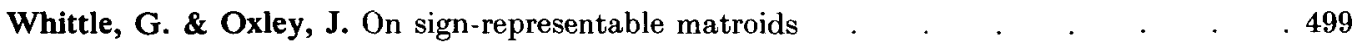

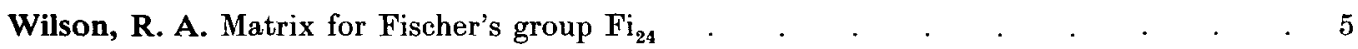

Wilson, S. M. J. \& Holland, D. Localization and class groups of module categories with exactness defects .

Yoneyama, T. \& Sugie, J. On the Liénard system which has no periodic solutions . . 413

Zamboni, L. \& Urbanski, M. On free actions of $\Lambda$-trees . . . . . . . . . . . . . 535

Zhang, B. \& Roach, G. F. Spectral representation and scattering theory for the wave equation with two unbounded media . . . . . . . . . . . . 423

Zimmermann, B. On a hyperbolic 3-manifold with some special properties. $\quad . \quad$. $\quad$. 87 


\section{THE PREPARATION OF MANUSCRIPTS}

\section{The attention of authors is particularly directed to the following requests.}

1. Papers should be typed, double-spaced, on one side of white paper (of which A4, 210 by $297 \mathrm{~mm}$, is a suitable size). The pages must be numbered. Margins of $30 \mathrm{~mm}$ should be left at the side, top and bottom of each page. Two clear copies should be sent.

A cover page should give the title, the author's name and institution, with the address at which mail is to be sent.

The title, while brief, must be informative (e.g. A new proof of the prime-number theorem, whereas Some applications of a theorem of $G$. H. Hardy would be useless).

The first paragraph or two should form a summary of the main theme of the paper, providing an abstract intelligible to mathematicians.

For a typescript to be accepted for publication, it must accord with the standard requirements of publishers, and be presented in a form in which the author's intentions regarding symbols ete. are clear to a printer (who is not a mathematician).

The following notes are intended to help the author in preparing the typescript. New authors may well enlist the help of senior colleagues, both as to the substance of their work and the details of setting it out correctly and attractively.

\section{Notation}

Notation should be chosen carefully so that mathematical operations are expressed with all possible neatness, to lighten the task of the compositor and to reduce the chance of error.

For instance $n_{k}$ ( $n$ sub $k$ ) is common usage. but avoid if possible using $c$ sub $n$ sub $k$. Fractions are generally best expressed by a solidus. Complicated exponentials like

should be shown in this and no other way.

$$
\exp \left\{z^{2} \sin \theta /\left(1+y^{2}\right)\right\}
$$

In the manuscript, italies, small capitals and capitals are specified by single, double and triple underlinings. Bold faced type is shown by wavy underlining; wavy will be printed wavy.

It helps if displayed equations or statements which will be quoted later are numbered in order on the right of their line. They can then be referred to by, for example, 'from (7)'.

The author must enable the printer (if necessary by pencilled notes in the margin) to distinguish between similar symbols such as $0, O \mathrm{o}, \mathrm{O}, 0 ; x, \mathrm{X}, \dot{x} ; \phi, \Phi, \varnothing ; 1,1 ; \varepsilon, \in ; \kappa, k$.

Greek letters can be denoted by Gk in the margin.

If an author wishes to mark the end of the proof of a theorem, the sign $\mathbf{m a y}$ be used.

Footnotes should be avoided.

\section{Diagrams}

It is extremely helpful if diagrams are drawn in Indian ink on white card, faintly blue or green-lined graph paper, or tracing cloth or paper. Symbols, legends and captions should be given on a transparent overlay. Each text figure must be numbered as Figure 1, Figure 2, .. and its intended position clearly indicated in the manuscript:

Figure 1 here

The author's name in pencil must be on all separate sheets of diagrams.

A figure is expensive to reproduce and should be included only when the subject matter demands it, or when it greatly clarifies the exposition.

The Society recognizes that some authors do not have the facilities for producing drawings of a sufficiently high standard to be reproduced directly and it is therefore willing to have such diagrams redrawn, provided that they are clear.

4. Tables

Tables should be numbered (above the table) and set out on separate sheets. Indicate the position of each in the text as for figures:

Table 3 here

\section{References}

References should be collected at the end of the paper numbered in alphabetical order of the authors' names. Titles of journals should be abbreviated as in Mathematical Reviews. The following examples show the preferred style for references to a paper in a journal, a paper in a proceedings volume, a book and an unpublished dissertation:

[1] J. F. Adams. On the non-existence of elements of Hopf invariant one. Ann. of Math. (2) 72 (1960), 20-104.

[2] M. P. Fourman and D. S. ScotT. Sheaves and logic. In Applications of Sheaves, Lecture Notes in Math. vol. 753 (Springer-Verlag, 1979), pp. 302-401

[3] P. T. Johnstone. Stone Spaces. Cambridge Studies in Advanced Math. no. 3 (Cambridge University Press, 1982)

[4] F. W. Lawvere. Functorial semantics of algebraic theories. Ph.D. thesis. Columbia University (1963). 


\section{Mathematical Proceedings of the Cambridge Philosophical Society \\ MPCPCO II3 (Pt 3) 449-638 (1993) 0305-004 I May I 993}

\section{CONTENTS}

SwINNTRTon-Dyme SiR P

RICKERT, J. H. Simultaneous rational approximations and related diophantine equations . . 461

Johnson, F. E. A. A rational invariant for certain infinite discrete groups . . . . . . 473

Hofmann, K. H. \& MorRIS, S. A. Generators on the are component of compact connected groups

RoBINson, P. L. Quasifree representations of Clifford algebras

OXLEY, J.\& WHITTLE, G. On sign-representable matroids

BAKER, J. W. \& LAU, A. T. Compact left ideal groups in semigroup compactification of locally compact groups.

AUINGER, K. The word problem for the bifree eombinatorial striet regular semigroup . .

URBANSKT, M \& ZAMBONI, I On free actions of $\Lambda$-trees.

HINKKANEN, A. Julia sets of rational functions uniformly perfect

Armitage, D. H., Gardiner, S. J. \& Netuka, I. Separation of points by elasses of harmonic functions.

Kamrya, S. On $H$-balls and canonical regions of loxodromic elements in complex hyperbolic space.

Brown, B. M., Kirby, V. G., Evans, W. D. \& Plum, M. Safe numerical bounds for the Titchmarsh-Weyl $m(\lambda)$-function

SzÜCs, A. Double point surfaces of smooth immersions $\dot{M}^{n} \rightarrow \mathbb{R}^{2 n-2}$

Kesten, H. Convergence in distribution of lightly trimmed and untrimmed sums are equivalent. 